\title{
Leveraging Design Principles to Inform the Next Generation of NASA Earth Satellites
}

\author{
By Joel P. Scott and Erin Urquhart
}

On September 23 and 24, 2020, NASA convened a virtual 2020 Plankton, Aerosol, Cloud, ocean Ecosystem (PACE) Applications Workshop to establish a transdisciplinary dialogue about how the PACE mission will support both applied research and societal needs, including managing water resources, safeguarding human health, supporting air quality monitoring, responding to a changing climate, and mitigating natural and anthropogenic disasters. The PACE mission is NASA's next great investment in Earth science, continuing its legacy of over 40 years of satellite ocean color measurements (Figure 1). Expected to launch no earlier than 2023, PACE will advance our Earth-observing and monitoring capabilities through hyperspectral imaging and multi-angle polarimetric observations of ocean, atmosphere, and land ecosystems. PACE will give us an unprecedented view of our home planet and will take Earth's pulse in new ways for years to come.

Applied science is a critical component of the PACE mission, and pre-launch application activities maximize the utility of NASA's investment in science, technol-

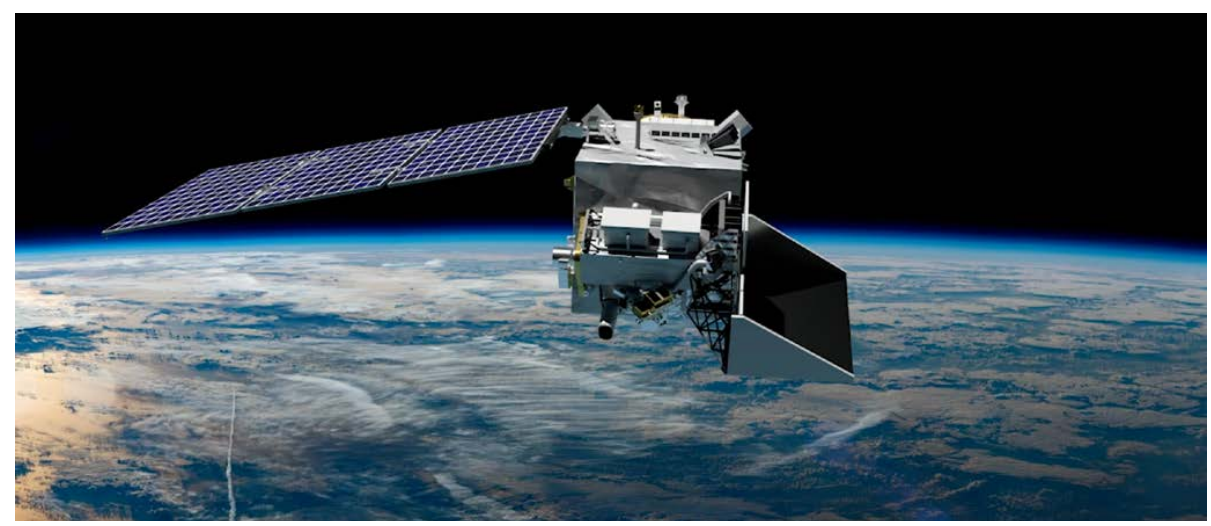

FIGURE 1. A rendering of the PACE spacecraft. Three instruments will fly on board: a hyperspectral imager and two multi-angle polarimeters. Credit: NASA Conceptual Image Laboratory, https://svs.gsfc.nasa.gov/12469 ogy, and innovation. Applied science is the use of scientific knowledge to advance society in support of stakeholder needs and to optimize decision-making processes. An integral component of applied research is design thinking-an iterative, problem-solving framework that integrates human perspectives, needs, and experiences at every step of the process. The 2020 PACE Application Workshop was the first in a series of annual PACE applied science events that will integrate design thinking principles into the mission, engaging stakeholders and leveraging user feedback to inform mission outcomes and deliverables.

Three key PACE design principlesdesirability, viability, and feasibility-were used to support the workshop's objectives and to underscore the importance of considering societal needs when designing the next generation of NASA Earthobserving satellites and developing innovative data products and data discovery tools. Three of the workshop's objectives were (1) to listen to and assess the current challenges that user communities face when working with satellite data, (2) to foster working partnerships between data producers and data users in support of open communication and collaboration, and (3) to identify potential applications of PACE data not currently being pursued. These objectives integrated desirability by assessing the needs of potential PACE data users and understanding their perceptions of satellite remote-sensing capabilities; viability by evaluating the utility of the PACE mission for health, economic, and environmental applications; and feasibility by assessing potential applied science projects within the scope of the PACE mission's anticipated performance and deliverables.

In support of the first two objectives, attendees participated throughout the workshop in live polling activities that were designed to provide an interactive forum to share their individual experiences with stakeholder interactions. Through these polling and free-response activities, attendees created a space where partnerships could be established, experiences could be shared, and ideas could be sparked to resolve challenges faced by different applied and stakeholder communities. As part of these activities, attendees ranked on a Likert scale of one (strongly disagree) to five (strongly agree) a series of statements about their stakeholder engagement experiences (Figure 2). The majority of participants agreed that not only have they identified their stakeholders (average rank: 3.7), but also that they understand their stakeholders' needs (3.4), consider their stakeholders' needs when designing projects (3.9), and actively collaborate with stakeholders (3.5).

Continuing on the theme of stakeholder engagement, a follow-up inter- 
active polling activity sought understanding of the major obstacles and challenges that often limit stakeholder and decisionmaker involvement in applied research. Workshop attendees submitted over 106 free-form responses that were then analyzed for common themes and plotted as a distribution of the whole (Figure 3 ). The most common obstacle mentioned was resource constraints when engaging stakeholders, and challenges identified included competing priorities, knowledge gaps, difficulties communicating science, and data limitations.

To conclude the co-creation activities focused on stakeholder engagement, workshop attendees were invited to discuss the benefits of stakeholder collaboration when conducting applied research projects. Workshop participants shared that when a user community's needs inform the design of data deliverables and products, the products themselves gain greater utility and often achieve broader adoption, perhaps even operationally, in support of societal needs. Some participants noted that stakeholder engagement can provide direct benefits such as acknowledgment, user feedback, and/or funding opportunities. The 2020 PACE Applications Workshop established that stakeholder engagement, while often challenging, has intrinsic merit and brings tangible benefits while building productive partnerships.

As a final example of how design thinking informed the 2020 PACE Applications Workshop, opportunities were provided throughout the event to contribute novel ideas for PACE applied science projects. Participants were encouraged to think of innovative ways that the mission's capabilities could be applied for the benefit of society and to increase the value and utility of the mission. The workshop provided a venue for the PACE applied and research communities to brainstorm these new application ideas with stakeholders and decision-makers. To initiate some of these conversations, a variety of existing PACE applied research projects were presented during two moderated

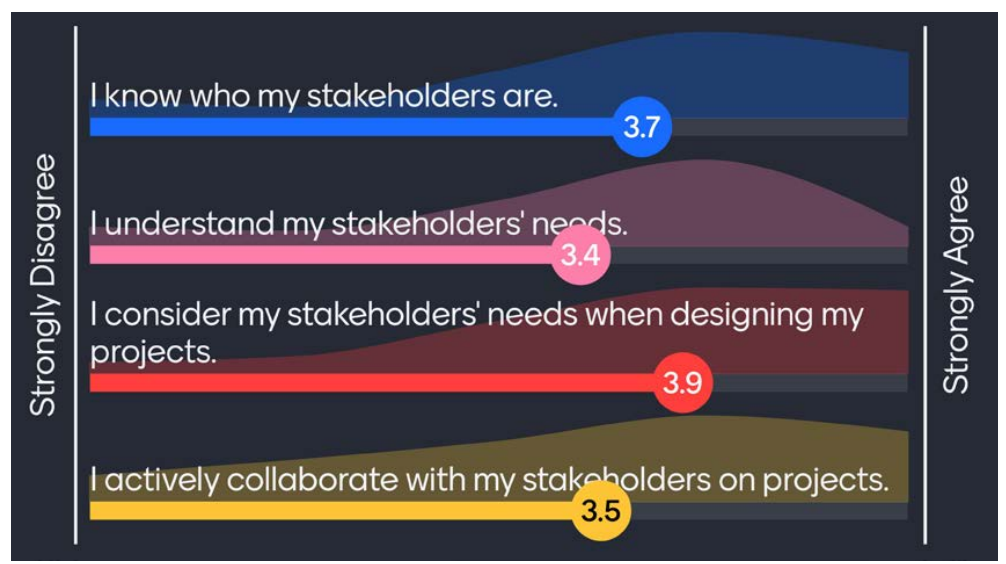

FIGURE 2. Workshop participants ranked, on a one-to-five Likert scale, a series of statements gauging their levels of stakeholder awareness and involvement via an interactive audience polling activity during the first day of the workshop.

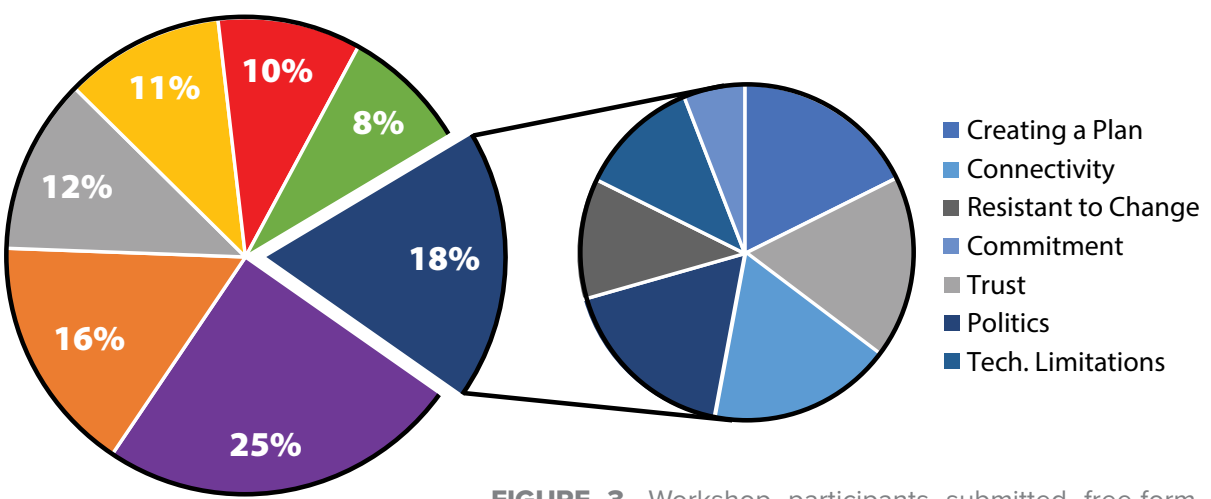

FIGURE 3. Workshop participants submitted free-form - Resource Constraints - Competing Priorities Knowledge Gap

Communication

- Data Limitations

- Understanding Needs

panel activities that highlighted water resource and air quality applications, as well as health and disaster response and mitigation projects. Workshop attendees shared their enthusiasm for a number of ideas that will be assessed for feasibility from PACE data and may potentially be targeted through future PACE applied research projects or applied science capacity-building initiatives.

The 2020 PACE Applications Workshop demonstrated the value of applied research at NASA, leveraging scientific knowledge to support real-world needs and develop practical tools that directly optimize decision-making processes. The event garnered positive feedback and enthusiasm for NASA science, data, and satellite technology and demon- strated that design principles are critically important when hosting an interactive transdisciplinary virtual event, as well as maximizing the utility of NASA Earth science investment. Workshop recordings and other resources may be found on the PACE Applications website (https://pace.oceansciences.org/ app_workshops.htm). @

\section{ACKNOWLEDGMENTS}

We would like to thank NASA and the NASA PACE mission for support to host this workshop and the PACE Applications Program.

\section{AUTHORS}

Joel P.Scott (joel.scott@nasa.gov) is PACE Applications Deputy Coordinator, NASA Goddard Space Flight Center, Greenbelt, MD, USA, and Science Applications International Corporation, Reston, VA, USA. Erin Urquhart is PACE Applications Coordinator, NASA Goddard Space Flight Center, Greenbelt, MD, USA, and Science Systems and Applications Inc., Lanham, MD, USA. 